J. Clin. Chem. Clin. Biochem.

Vol. 27, 1989, pp. 547-554

(C) 1989 Walter de Gruyter \& Co. Berlin - New York

\title{
Dipeptidyl-Aminopeptidase IV in einem klinischen Krankengut und bei Galaktosaminhepatitis der Ratte: Aktivität und Lektinaffinitätschromatographie in Serum und Leberplasmamembran
}

\author{
Von S. Eggstein \\ Chirurgische Universitätsklinik Freiburg, Abteilung Allgemeine Chirurgie mit Poliklinik \\ W. Kreisel, W. Gerok
}

Medizinische Universitätsklinik Freiburg Abteilung II und

M. Eggstein

Medizinische Universitätsklinik Tübingen Abteilung IV

(Eingegangen am 10. Februar/28. Juni 1989)

Zusammenfassung: Dipeptidyl-Aminopeptidase IV wurde im Serum von 378 Patienten untersucht. Bei Neoplasmata und Hepatitiden waren die Dipeptidyl-Aminopeptidase IV-Mittelwerte signifikant erhöht, nicht aber bei Lebercirrhose. Korrelationen bestanden zu den Cholestaseenzymen; nur bei den Neoplasmata zu Lactatdehydrogenase. Die Hauptkomponentenanalyse ordnete Dipeptidyl-Aminopeptidase IV keiner Enzymgruppe $\mathrm{zu}$, sondern stellte sie als eigenständigen Parameter dar.

An der Ratte wurde der Einfluß der Galaktosaminhepatitis auf die Dipeptidyl-Aminopeptidase IV-Aktivität und Glykosylierung in Serum und Leberplasmamembran untersucht. Im Verlauf der Hepatitis stieg die Aktivität der Dipeptidyl-Aminopeptidase IV in Serum und Leberplasmamembran an. Die Dipeptidyl-Aminopeptidase IV im Serum wies eine Wèizenkeim-Lektin nicht bindende Variante auf, welche in der Plasmamembran nicht vorhanden war. Die auf der Plasmamembran vorkommende Concanavalin A und WeizenkeimLektin bindende Glykosylierungsvariante trat im Verlauf der Galaktosaminhepatitis vermehrt im Serum auf. Dipeptidyl=Aminopeptidase IV im Serum des Menschen hatte das gleiche Lektinbindungsmuster wie die Dipeptidyl-Aminopeptidase IV der Plasmamenbran von Ratten. Die Lektinaffinitätschromatographie der Dipeptidyl-Aminopeptidase IV im Serum bei Lebercirrhose zeigte das gleiche Bindungsmuster wie bei Gesunden $(96 \%-98 \%$ Bindung an Concanavalin A und Weizenkeim-Lektin).

Dipeptidyl aminopeptidase IV in hospitalized patients and in galactosamine hepatitis of the rat: Activity and lectin affinity chromatography in serum and hepatic plasma membranes

Summary: The activity of dipeptidyl aminopeptidase IV was studied in the sera of 378 hospitalized patients. The mean activity of dipeptidyl aminopeptidase IV was elevated significantly in patients with neoplasmata and hepatitis, but not in patients with liver cirrhosis. Significant correlations $(p<0.001)$ existed with $\gamma$ glutamyl transferase, glutamate dehydrogenase, alkaline phosphatase and leucine aminopeptidase. A significant correlation with lactate dehydrogenase existed only in patients with neoplasmata. Principal component analysis, performed with aspartate aminotransferase, alanine aminotransferase, alkaline phosphatase, leucine aminopeptidase, lactate dehydrogenase and dipeptidyl aminopeptidase IV, revealed correlations between the activities 
of aspartate aminotransferase and alanine aminotransferase, and between alkaline phosphatase and leucine aminopeptidase, but neither dipeptidyl aminopeptidase IV nor lactate dehydrogenase showed any correlation with either of these two groups. In lectin affinity chromatography with concanavalin $A$ and wheat germ lectin sepharose, serum dipeptidyl aminopeptidase IV from liver cirrhosis patients showed the same binding pattern as that from healthy subjects.

The activity and glycosylation of dipeptidyl aminopeptidase IV in serum and hepatic plasma membranes was investigated in rats, following the induction of hepatitis with galactosamine. In the serum, dipeptidyl aminopeptidase IV activity was elevated as early as $6 \mathrm{~h}$ after galactosamine injection, and the elevated activity persisted until the $7^{\text {th }}$ day. At the same time dipeptidyl aminopeptidase IV activity was also elevated in the hepatic plasma membrane. Ninety eight percent of hepatic dipeptidyl aminopeptidase IV bound to concanavalin $\mathrm{A}$ as well as to wheat germ lectin and this value was unchanged during hepatitis. In the serum of control rats, $90 \%$ of dipeptidyl aminopeptidase IV bound to concanavalin A but only $39 \%$ to wheat germ lectin. During galactosamine hepatitis, the wheat germ lectin binding fraction of serum dipeptidyl aminopeptidase IV increased significantly $(p<0.001$ ) from $39 \%$ to $59 \%$. We suggest that the increased levels of dipeptidyl aminopeptidase IV in serum are derived from the hepatic plasma membrane, where dipeptidyl aminopeptidase IV accumulates during hepatitis.

Our data do not suggest a role for serum dipeptidyl aminopeptidase IV in clinical diagnosis.

\section{Einführung}

Dipeptidyl-Aminopeptidase IV (EC 3.4.14.5), ein Glykoprotein mit Exopeptidaseaktivität, ist in vielen Organen und Körperflüssigkeiten nachweisbar. Ihre physiologische Funktion liegt wahrscheinlich in der Spaltung von Fibrin (1) (antithrombotische Wirkung), Substance P (2) (Aktivierung, Gefäßregulation) und Hydrolyse Prolin-enthaltender Oligopeptide im Dünndarm (3) (Digestion). Der Dipeptidyl-Aminopeptidase IV-Nachweis hat diagnostische Bedeutung in der cytochemischen Differenzierung von T-lymphocytären Leukämien. Die diagnostische Wertigkeit der Dipeptidyl-Aminopeptidase IV-Bestimmung im Serum wird sehr kontrovers beurteilt. In der Literatur findet man widersprüchliche Angaben über den Anstieg der Dipeptidyl-Aminopeptidase IV-Aktivität im Serum bei Hepatitis, Lebercirrhose, Verschlußikterus, entzündlichen Darmerkrankungen, Herzinfarkt, Hypertonie und die Verminderung der Dipeptidyl-Aminopeptidase IV-Aktivität bei Magen- und -Pankreasneoplasmata und Leukosen (4-7).

Im Rahmen dieser Arbeit wurde die Dipeptidyl-Aminopeptidase IV-Aktivität im Serum in einem großen unselektierten Krankengut untersucht und zu Routinelaborparametern in Beziehung gesetzt. Da Glykoproteine eine große Variabilität der Glykosylierung aufweisen, wurde geprüft, ob die Sensitivität der Aktivitätsbestimmung im Serum durch Auftrennung der Glykosylierungsvarianten mit Lektinaffinitätschromatographie erhöht werden kann. Der Verlauf der Dipeptidyl-Aminopeptidase IV-Aktivität und die gleichzeitige Änderung des Glykosylierungsmusters in Serum und Leberplasmamembran wurde am Modell der Galaktosaminhepatitis der Ratte verfolgt.

\section{Material und Methoden}

In Seren von 378 unselektierten stationär im Universitätsklinikum Tübingen behandelten Patienten sowie 24 Kontrollpersonen wurde Aspartataminotransferase, Alaninaminotransferase, $\gamma$-Glutamyltransferase, alkalische Phosphatase, LeucinAminopeptidase, Glutamatdehydrogenase, Lactatdehydrogenase, Cholinesterase, Bilirubin, Serumeiweiß und DipeptidylAminopeptidase IV bestimmt. Im geșamten Krankengut und in einzelnen Diagnosegruppen wurden die Mittelwerte der Dipeptidyl-Aminopeptidase IV und Korrelationen zu den oben genannten Analyten errechnet. Mit Dipeptidyl=Aminopeptidase IV, Aspartataminotransferase, Alaninaminotransferase, alkalischer Phosphatase, Leucin-Aminopeptidase und Lactatdehydrogenase als Kenngrößen wurde eine Hauptkomponentenanalyse $(n=351)$ nach Quadratwurzeltransformation mit dem Programmpaket PAS, Proc. Factor (8) durchgeführt. An 6 Patienten mit Lebercirrhose und 9 Gesunden wurde die Lektinaffinitätschromatographie mit Concanavalin A-Sepharose und Weizenkeim-Lektin-Sepharose $6 \mathrm{MB}$ eingesetzt.

Nach intraperitonealer Injektion von Galaktosaminhydrochlorid $(375 \mathrm{mg} / \mathrm{kg}$ Körpergewicht gelöst in $0,8 \mathrm{ml}$ dest. Wasser, Fa. Roth, Karlsruhe) zur Induktion einer Hepatitis (9) bei männlichen Wistar-Ratten (Tierzucht İvanovas, Allgäu, Altromin ${ }^{\oplus}$, Wasser ad libitum) wurde nach $3,6,12 \mathrm{~h}$ und $2,3,4$, $5,6,7,8$ Tagen jeweils 4 Ratten durch Punktion der Vena cava Blut entnommen und Aspartataminotransferase, Alaninaminotransferase, alkalische Phosphatase, Glutamatdehydrogenase, Cholinesterase und Dipeptidyl-Aminopeptidase IV bestimmt. Die Lektinaffinitätșchromatographie wurde mit Serum und Leberplasmamembranen gesunder Ratten sowie 36 Ștunden und 8 Tage nach Hepatitisinduktion an jeweils 4 Tieren durchgeführt.

Aspartataminotransferase, Alaninaminotransferase, alkalische Phosphatase, Leucin-Aminopeptidase, Glutamatdehydrogenase, $\gamma$-Glutamyltransferase, Serumeiwei $\beta$, Bilirubin, Lactatdehydrogenase und Cholinesterase wurden nach Standardmethoden der Deutschen Gesellschaft für Klinische Chemie be: stimmt.

Die Dipeptidyl-Aminopeptidase IV-Aktivität im Serum wurde mit der Methode von Nagatsu (10) mit Glycyl-L-Prolin $=p=\mathrm{Ni}-$ troanilid-tosylat (Fa. Roth, Karlsiruhe) als Substrat bei $37^{\circ} \mathrm{C}$ und $405 \mathrm{~nm}$ bestimmt (Gilford Spectrophotometer $250 \mathrm{mit}$ Recorder, Gilford Instrument Lab. Inc., Oberlin, Ohio). Serum $(100, \mu \mathrm{l})$ wurde mit $100 \mu \mathrm{l}$ Substratlösung $(34,2 \mathrm{mmol} / \mathrm{l})$ und 
$1900 \mu \mathrm{l}$ Puffer A (pH 8.0, $0.1 \mathrm{~mol} / 1$ TRIS, 3,1 mmol/1 $\mathrm{NaN}_{3}$, $0.1 \mathrm{~mol} / 1 \mathrm{NaCl}, 1 \mathrm{mmol} / 1 \mathrm{CaCl}_{2}, 1 \mathrm{mmol} / 1 \mathrm{MgCl}_{2}, 5 \mathrm{~g} / 1$ Triton $X$ 100) 10 Minuten inkubiert und die Extinktionsänderung aufgezeichnet. Der Leerwert (100 $\mu$ l Substratlösung mit 2000 $\mu$ l Puffer A) wurde subtrahiert.

Leberplasmamembranen wurden wie von Bachmann (11) beschrieben präpariert. Nach Homogenisation im Glashomogenisator nach Polter-Elvehjem (Fa. Braun, Melsungen) mit 2000 $\mu l$ Medium (1.0 mmol/1 $\mathrm{NaHCO}_{3}, 0.5 \mathrm{mmol} / 1 \mathrm{CaCl}_{2}$ ) wurde Dipeptidyl-Aminopeptidase IV in folgendem Ansatz bestimmt: $20 \mu$ l Plasmamembranhomogenat, $930 \mu \mathrm{l}$ Puffer A, $50 \mu \mathrm{l} \mathrm{Sub-}$ stratlösung.

Die Proteinbestimmung erfolgte nach Lowry et al. (12).

Für die Lektinaffinitätschromatographie wurden jeweils $1 \mathrm{ml}$ Serum oder Tritonextrakt aus Plasmamembranen (hergestellt nach l.c. (13)) auf $12 \mathrm{ml}$ Lektingel (Concanavalin A Sepharose und Weizenkeim-Lektin $6 \mathrm{MB}$, Pharmacia Fine Chemicals, Uppsala) aufgetragen und nach 15 minütigem Einwirken mit $150 \mathrm{ml}$ Puffer A bei $22^{\circ} \mathrm{C}$ portionsweise eluiert. Die lektingebundene Dipeptidyl-Aminopeptidase IV wurde mit $4 \mathrm{~g} / 1 \mathrm{~N}$ Acetylglucosamin (Sigma, St. Louis) in Puffer A für Weizenkeim-Lektin und $4 \mathrm{~g} / \mathrm{l} \alpha$-Methylmannopyranosid (Fa. Roth, Karlsruhe) für Concanavalin A eluiert. Für die DipeptidylAminopeptidase IV-Bestimmung wurden $2000 \mu \mathrm{l}$ Eluat mit 100 $\mu \mathrm{l}$ Substratlösung inkubiert.

Zur statistischen Auswertung diente der t-Test und die FischerTransformation.

\section{Ergebnisse}

Enzymprofil im Serum bei Galaktosaminhepatitis (Abb. 1)

Bereits $6 \mathrm{~h}$ nach Galaktosaminapplikation war die Dipeptidyl-Aminopeptidase IV-Aktivität im Serum mit $34 \mathrm{U} / 1$ signifikant $(\mathrm{p}<0,001)$ gegenüber der gesunden Kontrollgruppe (20 U/1) erhöht. Die maximale Dipeptidyl-Aminopeptidase IV-Aktivität am 2. Tag war $7 \mathrm{mal}$ höher als der Ausgangswert, welcher nach 8 Tagen wieder erreicht war. Aspartat- und Alaninaminotransferase ẅaren bereits nach $24 \mathrm{~h}$ maximal erhöht (Aspartataminotransferase auf das 36fache) und nach 4 Tagen wieder im Referenzbereich. Die Glutamatdehydrogenase war nach $24 \mathrm{~h}$ auf dem Höchstwert mit dem 352făchen des Ausgangswertes anbelangt und zwar nach 4 Tagen wieder auf Referenzwerte abgefallen. Die Cholinesterase sank am 2. Tag auf $2 / 3$ des Ausgangswertes und erreichte nach 5 Tagen wieder normale Wẹte. Die höchste Aktivität der alkalischen Phosphatase wurde nach 2 Tagen gemessen. Sie sank nach 6 Tagen wieder auf normale Werte.

Im zeitlichen Verlauf der Galaktosaminhepatitis korrelierten die Aktivitäten der Dipeptidyl-Aminopeptidase IV im Serum hochsignifikant $(p<0,001)$ mit den Werten der alkalischen Phosphatase $(r=0,88)$. Weitere signifikante Korrelationen bestanden nicht.
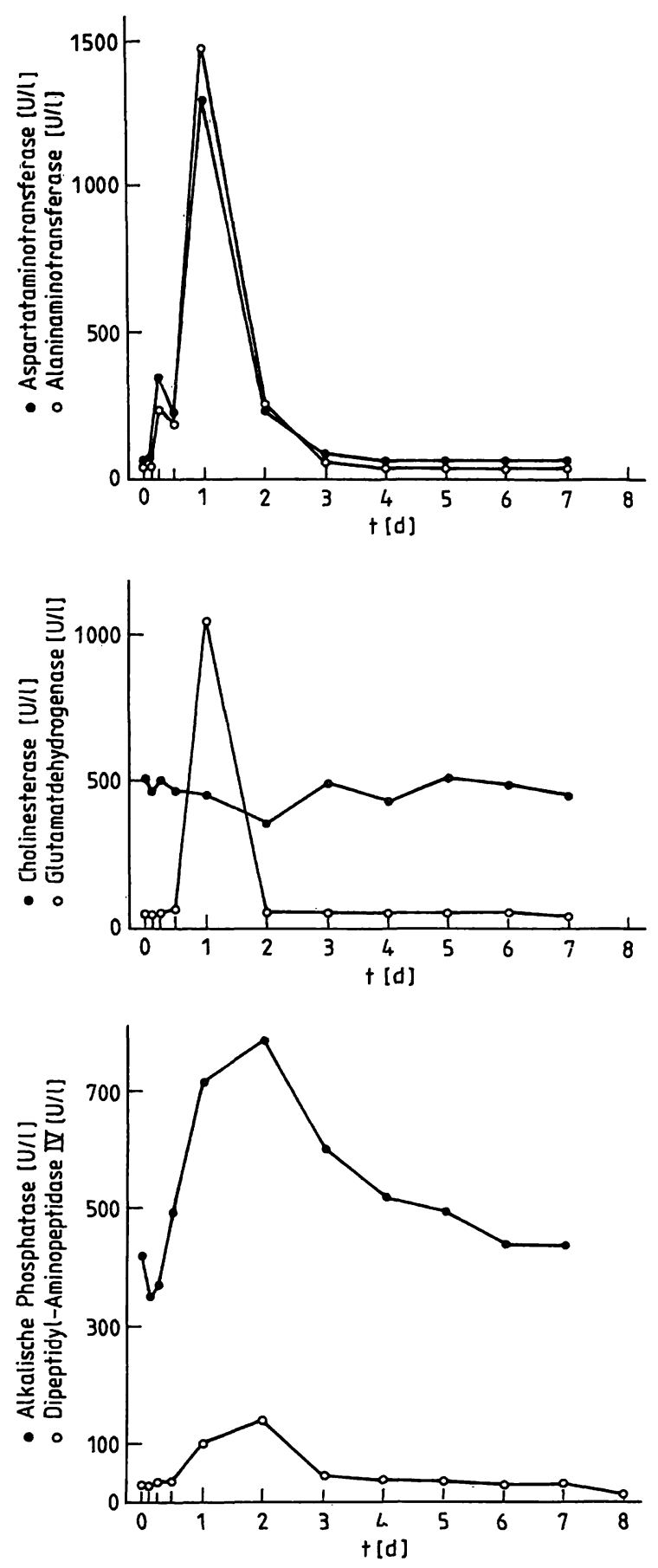

Fig. 1. Serumenzymprofil der Galaktosaminhepatitis: Mittelwerte von jeweils 4 Tieren

Dipeptidyl-Aminopeptidase IV-Aktivität in der Leberplasmamembran

Die Dipeptidyl-Aminopeptidase IV-Aktivität im Homogenat der Leberplasmamembranen von 4 Ratten stieg $36 \mathrm{~h}$ nach Galaktosamininjektion auf das 1,5fache des Ausgangswertes. Diese Aktivitätszunahme war signifikant $(\mathrm{p}<0,01)$. Nach 8 Tagen erreichte die Aktivität der Dipeptidyl-Aminopeptidase IV in Plasmamembranen wieder Normalwerte (Tab. 1). 
Tab. 1. Aktivität und Lektinbindung der Dipeptidyl-Aminopeptidase IV in Leberplasmamembranen bei Galaktosamin(Gal- $\mathrm{N}$ )Hepatitis der Ratte

Mittlere Dipeptidyl-Aminopeptidase IV-Aktivität in Units pro mg Protein im Plasmamembranhomogenat.

Lektinbindung der Dipeptidyl-Aminopeptidase IV in Tritonüberstand von Plasmamembranen in Prozent.

\begin{tabular}{|c|c|c|c|}
\hline & Vor Gal-N-Injektion & $\begin{array}{l}36 \text { h nach Gal-N- } \\
\text { Injektion }\end{array}$ & $\begin{array}{l}8 \text { Tage nach Gal-N- } \\
\text { Injektion }\end{array}$ \\
\hline $\begin{array}{l}\text { Dipeptidyl-Aminopeptidase IV (U/mg) } \\
\bar{x} \pm s(n=4)\end{array}$ & $0,44 \pm 0,08$ & $0,68 \pm 0,13$ & ; $\quad 0,36 \pm 0,07$ \\
\hline $\begin{array}{l}\text { Bindung an Concanavalin A (\%) } \\
\bar{x} \pm s(n=6)\end{array}$ & $98 \pm 2$ & $97 \pm 4$ & $99 \pm 2$ \\
\hline $\begin{array}{l}\text { Bindung an Weizenkeim-Lektin (\%) } \\
\bar{x} \pm s\end{array}$ & $98 \pm 4$ & $98 \pm 2$ & $97 \pm 3$ \\
\hline
\end{tabular}

\section{Lektinaffinitätschromatographie}

Die Lektinaffinitätschromatographie an 6 Ratten vor und $36 \mathrm{~h}$ nach Galaktosamininjektion ergab eine mit dem Anstieg der Dipeptidyl-Aminopeptidase IV im Serum einhergehende Änderung des Lektinbindungsverhaltens: Die Bindung der Dipeptidyl-Aminopeptidase IV im Serum an Weizenkeim-Lektin stieg von $39 \%$ bei unbehandelten Ratten auf $59 \%$ bei HepatitisTieren. Die Bindung an Concanavalin A blieb auch nach Galaktosamininjektion im Serum unverändert über $90 \%$ (Tab. 2).

Tab. 2. Lektinbindung der Dipeptidyl-Aminopeptidase IV im Serum bei Galaktosamin(Gal-N)-Hepatitis

\begin{tabular}{lll}
\hline & $\begin{array}{l}\text { Vor Gal-N- } \\
\text { Injektion }\end{array}$ & $\begin{array}{l}36 \mathrm{~h} \\
\text { nach Gal-N- } \\
\text { Injektion }\end{array}$ \\
\hline $\begin{array}{l}\text { Bindung an Concanavalin A } \\
(\%) \overline{\mathrm{x}} \pm \mathrm{s}\end{array}$ & $93 \pm 9$ & $96 \pm 3$ \\
$\begin{array}{l}\text { Bindung an Weizenkeim-Lektin } \\
(\%) \overline{\mathrm{x}} \pm \mathrm{s}\end{array}$ & $39 \pm 7$ & $59 \pm 5$ \\
$\begin{array}{l}\text { Dipeptidyl-Aminopeptidase IV- } \\
\text { Aktivität }(\mathrm{U} / \mathrm{l}) \overline{\mathrm{x}} \pm \mathrm{s}\end{array}$ & $20 \pm 5$ & $98 \pm 24$ \\
\hline
\end{tabular}

Dipeptidyl-Aminopeptidase IV im Tritonextrakt von Leberplasmamembranen band zu $98 \%$ an Concanavalin A und Weizenkeim-Lektin. Der Aktivitätsanstieg nach Galaktosamininjektion brachte keine Änderung des Bindungsverhaltens der Dipeptidyl-Aminopeptidase IV von Plasmamembranen mit sich (Tab. 1).

\section{Klinische Studie}

Der Dipeptidyl-Aminopeptidase IV-Mittelwert bei 24 Gesunden lag bei $24 \mathrm{U} / 1(\mathrm{~s}=4,4 \mathrm{U} / 1)$.
Im unselektierten Gesamtkrankengut war der Dipeptidyl-Aminopeptidase IV-Mittelwert mit 31,1 U/1 $(s=14,5 \mathrm{U} / \mathrm{l})$ signifikant $(\mathrm{p}<0,001)$ erhöht.

Hochsignifikante Korrelationen $(p<0,001)$ fanden sich zwischen Dipeptidyl-Aminopeptidase IV und $\gamma$ Glutamyltransferase $(r=0,47, n=218)$, Glutamatdehydrogenase $(r=0,36, n=278)$, alkalischer Phosphatase $(r=0,29, n=366)$ und Leucin-Aminopeptidase $(r=0,44, n=367)$.

Mit Aspartataminotransferase, Alaninaminotransferase, alkalischer Phosphatase, Leucin-Aminopeptidase, Lactatdehydrogenase und Dipeptidyl-Aminopeptidase IV $(n=351)$ wurde eine Hauptkomponentenanalyse durchgeführt. Mit 3 unabhängigen Fak-

Tab. 3. Hauptkomponentenanalyse mit sechs Enzymen im Serum im klinischen Krankengut (s. Abb. 2):

\begin{tabular}{lccc}
\hline Factor Pattern: & Faktor 1 & Faktor 2 & Faktor 3 \\
\hline $\begin{array}{l}\text { Dipeptidyl-Amino- } \\
\text { peptidase IV }\end{array}$ & 0,49085 & $-0,67774$ & $-0,15106$ \\
$\begin{array}{l}\text { Aspartatamino- } \\
\text { transferase }\end{array}$ & 0,82911 & 0,2845 & $-0,32985$ \\
$\begin{array}{l}\text { Alaninamino- } \\
\text { transferase }\end{array}$ & 0,76743 & 0,11482 & $-0,53106$ \\
$\begin{array}{l}\text { Alkalische Phos- } \\
\text { phatase }\end{array}$ & 0,67517 & $-0,17366$ & 0,61336 \\
$\begin{array}{l}\text { Lactatdehydro- } \\
\text { genase }\end{array}$ & 0,45865 & 0,69970 & 0,31022 \\
$\begin{array}{l}\text { Leucin-Amino- } \\
\text { peptidase }\end{array}$ & 0,81680 & $-0,23874$ & 0,24336 \\
\end{tabular}

Varianz:

Faktor 1 Faktor 2 Faktor 3

$2,850675 \quad 1,130194 \quad 0,945319$


toren konnten $82 \%$ der Varianz der 6 Kenngrößen erklärt werden. Das Korrelationsmuster der 3 Faktoren mit den 6 Kenngrößen (Tab. 3) wird in Abbildung 2 deutlich: Aspartataminotransferase liegt mit Alaninaminotransferase und alkalische Phosphatase mit Leucin-Aminopeptidase im gleichen Quadranten des dreidimensionalen Koordinatensystems entsprechend ihrer mathematischen Korrelation mit den 3 Faktoren. Lactatdehydrogenase und Dipeptidyl-Aminopeptidase IV sind isoliert in jeweils eigenen Quadranten lokalisiert.

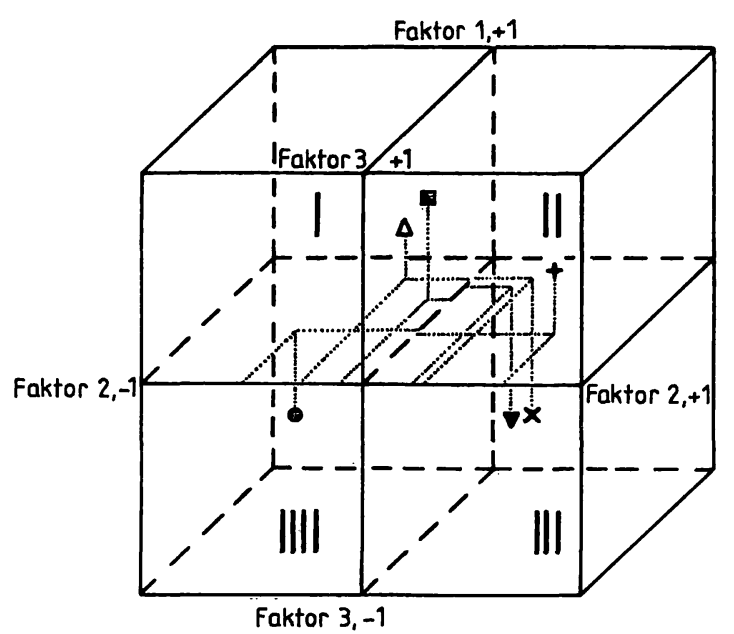

Fig. 2. Graphische Darstellung der Hauptkomponentenanalyse:

Die Koordinaten in der dreidimensionalen Darstellung entsprechen den drei Faktoren aus Tabelle 3. Die Position der Enzyme wird durch ihre Korrelation mit den Faktoren bestimmt.

- Dipeptidyl=Aminopeptidase IV

$x$ Aspartataminotransferase

$\nabla$ Alaninaminotransferase

a Alkalische Phosphatase

$\triangle$ Leucin-Aminopéptidase

+ Lactatdehydrogenase

\section{Diagnosegruppen}

Aus dem Gesamtkrankengut wurden folgende Dia= gnosegruppen selektiert (Tab. 4):

\section{Leukosen $(\mathrm{n}=26)$}

Der Dipeptidyl-Aminopeptidase IV-Mittelwert (27,7 $\mathrm{U} / 1, \mathrm{~s}=18,7)$ dieser Gruppe unterschied sich nicht signifikant von den Gesunden und auch nicht vom Gesamtkrankengut. Signifikante Korrelationen zu anderen Analyten bestanden nicht.

\section{Neoplasmata $(\mathrm{n}=31)$}

Den größten Anteil stellten Mamma- $(n=12)$ und Bronchialcarcinome $(n=6)$. Der Dipeptidyl-Aminopeptidase IV-Mittelwert lag mit 33,1 $\mathrm{U} / 1(\mathrm{~s}=18,3)$ signifikant $(\mathrm{p}<0,01)$ über dem der Gesunden. Nur in dieser Gruppe korrelierte Dipeptidyl-Aminopeptidase IV signifikant mit Lactatdehydrogenase $(\mathrm{p}<0,01, \mathrm{r}=0,47)$.

\section{Virushepatitis $(\mathrm{n}=14)$}

Hier fanden wir die höchsten Dipeptidyl-Aminopeptidase IV-Werte, im Mittel 46,6 U/1 $(s=14,2)$. Dieser Mittelwert war signifikant $(p<0,001)$ höher als bei den Gesunden und den Nichthepatitiskranken des Gesamtkrankengutes. Signifikante Korrelationen zu anderen Enzymen im Serum bestanden nicht.

\section{Lebercirrhose $(\mathrm{n}=17)$}

Der Dipeptidyl-Aminopeptidase IV-Mittelwert war mit $34 \mathrm{U} / 1$ und der hohen Standardabweichung von 28,7 U/1 nicht signifikant erhöht gegenüber dem Referenzkollektiv. Signifikante Korrelationen zu anderen Enzymen im Serum lagen nicht vor.

Lektinaffinitätschromatographie der Dipeptidyl-Aminopeptidase IV im Serum bei Lebercirrhose und Gesunden

98\% der Dipeptidyl-Aminopeptidase IV aus dem Serum von Gesunden $(n=6)$ banden an Concanavalin A, $96 \%$ der Dipeptidyl-Aminopeptidase IV banden an Weizenkeim-Lektin.

Dieses Bindungsmuster blieb unverändert bei $9 \mathrm{~Pa}$ tienten mit Lebercirrhose (Tab. 5).

Tab. 4. Diagnosegruppen im Überblick:

\begin{tabular}{llllll}
\hline & Leukosen & Neoplasmata & Hepatitis & Lebercirrhose & Gesunde \\
\hline $\mathrm{n}$ & 26 & 31 & 14 & 17 & 24 \\
$\begin{array}{l}\text { Dipeptidyl-Aminopeptidase IV (U/1) } \\
\overline{\mathrm{x}} \pm \mathrm{s}\end{array}$ & $27,7 \pm 18,7$ & $33,1 \pm 18,3$ & $46,6 \pm 14,2$ & $34,0 \pm 28,7$ & $24,0 \pm 4,4$ \\
Korrelation* mit & - & $\begin{array}{l}\text { Lactat- } \\
\text { dehydrogenase }\end{array}$ & - & - & - \\
\hline
\end{tabular}

* signifikante Korrelation $(\mathrm{p}<0,01)$ 
Tab. 5. Lektinbindung von Dipeptidyl-Aminopeptidase IV im Serum des Menschen bei Lebercirrhose und Gesunden:

\begin{tabular}{lcc}
\hline & Gesunde & $\begin{array}{l}\text { Leber- } \\
\text { cirrhose }\end{array}$ \\
\hline $\mathrm{n}$ & 6 & 9 \\
Bindung an Concanavalin A (\%) & $98 \pm 2$ & $98 \pm 1$ \\
$\overline{\mathbf{x}} \pm \mathrm{s}$ & & $97 \pm 2$ \\
$\begin{array}{l}\text { Bindung an Weizenkeim-Lektin (\%) } \\
\overline{\mathbf{x}} \pm \mathrm{s}\end{array}$ & $96 \pm 5$ & \\
$\begin{array}{l}\text { Dipeptidyl-Aminopeptidase } \\
\text { IV-Aktivität }(\mathrm{U} / \mathrm{l}) \overline{\mathrm{x}} \pm \mathrm{s}\end{array}$ & $29 \pm 15$ & $32 \pm 17$ \\
\hline
\end{tabular}

\section{Diskussion}

Am Modell der Galaktosaminhepatitis der Ratte sollten die Auswirkungen der Hepatitis auf ein hepatozelluläres Glykoprotein, die Dipeptidyl-Aminopeptidase IV, untersucht werden. Trotz vieler widersprüchlicher Angaben über die Aktivität der DipeptidylAminopeptidase IV im Serum bei hepatobiliären Erkrankungen $(4,5)$ gibt es bisher keine experimentellen Untersuchungen zum zeitlichen Verlauf der Aktivität im Serum nach Einwirken der Noxe, über die Aktivität der Dipeptidyl-Aminopeptidase IV in der Leberzellmembran und über die Glykosylierung bzw. Änderung der Glykosylierung im Verlauf der Erkrankung.

Die Amplitude des Dipeptidyl-Aminopeptidase IVAnstieges im Verlauf der Galaktosaminhepatitis ist mit dem 7fachen zwar gering im Vergleich mit den Transaminasen und Glutamatdehydrogenase, aber die Dipeptidyl-Aminopeptidase IV war das am frühesten (6 h) und am längsten (8 Tage) erhöhte Enzym. Dieser Verlauf ist der alkalischen Phosphatase ähnlich, welche auch vornehmlich mikrosomaler Herkunft und gallekanalikulär lokalisiert ist $(14,15)$. Dies kommt in der hochsignifikanten Korrelation $(p<0,001)$ beider Enzyme zum Ausdruck.

Gleichzeitig mit dem Anstieg der Dipeptidyl-Aminopeptidase IV-Aktivität im Serum war auch die Dipeptidyl-Aminopeptidase IV-Aktivität in der Leberplasmamembran erhöht.

Die Lektinaffinitätschromatographie zeigte Unterschiede der Glykosylierung von Dipeptidyl-Aminopeptidase IV im Serum und Dipeptidyl-Aminopeptidase IV der Plasmamembran: Während in der Plasmamembran die Dipeptidyl-Aminopeptidase IV vollständig an Concanavalin A und Weizenkeim-Lektin bindet, gibt es im Serum zusätzlich eine WeizenkeimLektin nicht bindende Variante. Diese Glykosylierungsvariante der Dipeptidyl-Aminopeptidase IV trägt $59 \%$ zur Gesamtaktivität bei. Sie könnte entweder durch Einwirken von Glykosidasen aus membranärer Dipeptidyl-Aminopeptidase IV entstehen oder aus anderen Organen stammen, z. B. dem Darm, wo Erickson et al. (16) ebenfalls Weizenkeim-Lektin nicht bindende Dipeptidyl-Aminopeptidase IV fanden. Im Verlauf der Galaktosaminhepatitis steigt der Anteil der Weizenkeim-Lektin bindenden DipeptidylAminopeptidase IV im Serum, also der Variante, die in der Plasmamembran der Leber vorhanden ist. Gleichzeitig steigt in der Plasmamembran die Aktivität der Dipeptidyl-Aminopeptidase IV ohne Änderung des Glykosylierungsmusters.

Wir schließen daraus, daßૂ Dipeptidyl-Aminopeptidase IV bei der Galaktosaminhepatitis vermehrt aus den Mikrosomen in die Plasmamembran eingebaut und von dort ins Serum abgegeben wird. Da Galaktosamin durch Manipulation des hepatozellulären Uracilnucleotid-Stoffwechsels (Akkumulation von Uridin-diphosphat-galaktosamin, Mangel an Uridintriphosphat) die Proteinsynthese inhibiert (9), ist nicht anzunehmen, daß eine gesteigerte Neusynthese von Dipeptidyl-Aminopeptidase IV stattfindet. Ursächlich für die vermehrte Dipeptidyl-Aminopeptidase IV-Ak= tivität in der Plasmamembran könnte aber eine Störung des vesikelvermittelten intrazellulären Transportes sein, welche die Interiorisation membranständiger Dipeptidyl-Aminopeptidase IV inhibiert.

Ziel der klinischen Studie war, Dipeptidyl-Aminopeptidase IV in ihrer Beziehung zu diagnostisch bewährten Routineanalyten darzustellen und zu prüfen, ob Veränderungen der Dipeptidyl-Aminopeptidase IVAktivität im Serum bestimmten Krankheitsgruppen zuzuordnen sind.

Der Dipeptidyl-Aminopeptidase IV-Mittelwert des unselektierten Gesamtkrankengutes lag signifikant über dem der Gesunden. Das war bei der Vielzahl von Erkrankungen, die mit erhöhten Dipeptidyl-Aminopeptidase IV-Werten einhergehen sollen, zu erwarten. Hopsu-Havu et al. (17) errechneten bei $286 \mathrm{~Pa}$ tienten keinen gegenüber Gesunden erhöhten Dipeptidyl-Aminopeptidase IV-Mittelwert, obwohl sie mit 22,6 U/1 annähernd den gleichen Dipeptidyl-Aminopeptidase IV-Mittelwert bei Gesunden wie. wir (24 U/1) fanden. Diese Unterschiede sind wahrscheinlich in der unterschiedlichên Zusammensetzung des Krankengutes begründet.

Im Gesamtkrankengut bestanden hochsignifikante Korrelationen zwischen Dipeptidyl-Aminopeptidase IV und alkalischer Phosphatase, Leucin-Aminopeptidase, $\gamma$-Glutamyltransferase und Glutamatdehydrogenase $(p<0,001)$. Damit könntè Dipeptidyl-Aminopeptidase IV zu den Cholestase-anzeigenden En- 
zymen gehören, was die enge Korrelation zur alkalischen Phosphatase bei der Galaktosaminhepatitis zu bestätigen scheint.

Diese Korrelationen sind auffälligerweise bei Hepatitiden und Lebercirrhosen nicht nachweisbar. Dafür besteht in der Diagnosegruppe Neoplasmata neben einem erhöhten Dipeptidyl-Aminopeptidase IV-Mittelwert eine Korrelation mit Lactatdehydrogenase, die sich im Gesamtkrankengut nicht nachweisen läßt. Möglicherweise wird also auch Dipeptidyl-Aminopeptidase IV als unspezifisches Zeichen des Zellzerfalls aus Tumorgewebe wie Lactatdehydrogenase freigesetzt oder gelangt aufgrund struktureller Veränderungen der Tumorzellmembran vermehrt ins Serum, wie es beim Morris-Hepatom beschrieben wurde (18).

Signifikant erhöhte Dipeptidyl-Aminopeptidase IVWerte bestanden, den Befunden des Tiermodells entsprechend, bei Hepatitiden, wo die höchsten Dipeptidyl-Aminopeptidase IV-Werte gefunden wurden. Aber auch hier war die Amplitude des DipeptidylAminopeptidase IV-Anstiegs(Verdoppelung), verglichen mit den Transaminasen, gering.

Bei Leukosen fanden wir im Gegensatz zu Literaturangaben (6) keine erniedrigten Dipeptidyl-Aminopeptidase IV-Werte.

Mit der Faktorenanalyse sollte die Beziehung der Dipeptidyl-Aminopeptidase IV zu den in der Routineanalytik bestimmten Enzymen Aspartataminotransferase, Alaninaminotransferase, alkalische Phosphatase, Leucin-Aminopeptidase und Glutamatdehydrogenase weiter geklärt werden. Dieses Verfahren (Abb. 2) zeigt die Zusammenhänge und Abhängigkeiten zwischen den 6 Enzymen durch Reduktion auf 3 mathematischen Faktoren. Das mathematische Modell verdeutlicht die klinisch bekannte Korrelation zwischen Aspartat-/Alaninaminotransferase und alkalischer Phosphatase/Leucin-Aminopeptidase genauso wie die
Sonderstellung der Lactatdehydrogenase. Keinem dieser Enzyme wird jedoch Dipeptidyl-Aminopeptidase IV zugeordnet. Abbildung 2 veranschaulicht die Beziehung der Enzyme zu den drei Faktoren, welche als Koordinaten im dreidimensionalen Koordinatensystem dargestellt sind.

Einen geringfügigen Dipeptidyl-Aminopeptidase IVAktivitätsanstieg findet man also bei einer Vielzahl von Erkrankungen, ohne Spezifität für eine Diagnosegruppe. Auch wenn Korrelationen zu den CholestaseEnzymen bestehen, gehört Dipeptidyl-Aminopeptidase IV nach dem Ergebnis der Hauptkomponentenanalyse nicht dieser Gruppe an.

Der Dipeptidyl-Aminopeptidase IV-Mittelwert bei Lebercirrhose lag zwar über dem Wert Gesunder, war aber aufgrund der hohen Standardabweichung nicht signifikant erhöht. Mit Hilfe der Lektinaffinitätschromatographie untersuchten wir, ob sich signifikante Änderungen der Glykosylierung ähnlich wie bei der Galaktosaminhepatitis nachweisen und damit vielleicht Spezifität und Sensitivität der Dipeptidyl-Aminopeptidase IV-Bestimmung erhöhen ließen. Im Serum von Gesunden als auch von Lebercirrhose-Kranken fand sich jedoch als einziger Typ Concanavalin A und Weizenkeim-Lektin bindende Dipeptidyl-Aminopeptidase IV. Weitere Untersuchungen mit Hilfe anderer Lektine müssen zeigen, ob neben speziesspezifischen Unterschieden in der Glykosylierung der Dipeptidyl-Aminopeptidase IV im Serum auch klinisch relevante, krankheitsspezifische Glykosylierungsvarianten existieren.

\section{Danksagung}

Wir danken Herrn Dr. Maulbetsch, Medizinische Universitätsklinik Tübingen, für die statistischen Berechnungen und Herrn Dr. $M a u$, Institut für Biomathematik der Universität Tübingen, für die Hauptkomponentenanalyse.

\section{Literatur}

1. Mentlein, R. \& Heymann, E. (1982) Dipeptidyl peptidase IV inhibits the polymerisation of fibrịn monomers. Arch. Biochem. Biophys. 217, $748 \div 750$.

2. Heymann, E. \& Mentlein, R. (1978) Liver dipeptidylaminopeptidase IV hydrolyses substance P. FEBS-Letters 91 , $360-364$.

3. Morita, A., Chung, Y., Freemann, H., Erickson, R., Sleisenger, M. \& Kim, Y. (1982) Intestinal assimilation of a proline containing tetrapeptide. J. Clin. Invest. 72, 610616.

4. Hino, M., Nagatsu, T., Kakumo, S., Okuyama, S., Yoshii, Y. \& Nagatsu, J. (1975) Glycyl-prolyl-betạ-naphthylamidase activity in human serum. Clin. Chim. Acta 62, 5-11.

5. Hutchinson, D., Halliwell, R., Lockard, I. \& Parke, D. (1981) Glycyl-proline-p-nitroanilidase in hepatobiliary disease. Clin. Chim. Acta 109, 83-89.

6. Fujita, K., Hirano, M., Tokunoga, K., Nagatsu, J., Nagatsu, T. \& Sakakibara, S. (1977) Serum glycyl-prolin-pnitroanilidase in blood cancers. Clin. Chim. Acta 81, 215217.

7. Burckhard, U., Klagge, M. \& Haftstein, M. (1978) Serum DPP IV-Aktivität bei Erkrankungen innerer Organe. $Z$. Klin. Med. 245-249.

8. Barr, A., Goodnight, J., Sall, J., Blair, W. \& Chilko, D. (1979) SAS user's guide. Edition: Raleigh, North Carolina: SAS Institute Inc.

J. Clin. Chem. Clin. Biochem. / Vol. 27, 1989/ No. 9 
9. Keppler, D., Pausch, J. \& Decker, K. (1974) Selective uridine triphosphate deficiency induced by $D$-galactosamine in liver and reversed by pyrimidine nucleotide precursors. J. Biol. Chem. 249, 211-216.

10. Nagatsu, T., Hino, M. \& Fuyamada, H. (1976) New chromogenic substrates for $x$-prolyl dipeptidyl-aminopeptidase. Anal. Biochem. 74, 466-470.

11. Bachmann, W., Harms, E., Hassels, B., Henninger, H. \& Reutter, W. (1977) Studies on rat liver plasma membrane. Altered protein and phospholipid metabolism after injection of D-galactosamine. Biochem. J. 166, 455-462.

12. Lowry, O., Rosebourgh, L., Farr, A. \& Randall, R. (1951) Protein measurement with the Folin reagent. J. Biol. Chem. 193, 265-275.

13. Kreisel, W., Heussner, R., Volk, B., Büchsel, R., Reutter, W. \& Gerok, W. (1982) Identification of the $110000 M_{r}$ glycoprotein isolated from rat liver plasma membrane as dipeptidylaminopeptidase IV. FEBS-Letters 147, 85-88.
14. Kreisel, W., Büchsel, R., Volk, B., Reutter, W: \& Gerok, W. (1982) Turnover of plasma membrane glycoproteins. In: Structural carbohydrates of the liver. MTP Press Limited pp. $51-62$.

15. Hopsu-Havu, V. \& Sarimo, S. (1967) Purification and characterisation of an aminopeptidase hydrolyzing glycyl-proline-naphthylamide. Hoppe Seỹler's Z. Physiol. Chem. 348, 1540-1550.

16. Erickson, R. \& Kim, Y. (1983) Interaction of purified brushborder membrane aminopeptidase $\mathrm{N}$ and dipeptidylpeptidase IV with lectin-sepharose derivates. Biochim. Biophys. Acta 743, 37 = 42 .

17. Hopsu-Havu, V., Jansen, C. \& Järvinen, M. (1970) A human serum aminopeptidase capable of splitting bonds involving proline. Clin. Chim. Acta 28, 25-36.

18. Hanski, C.., Z̈immer, T., Gossrau, R. \& Reutter, W. (1986) Increased activity of dipeptidyl peptidase 4 in serum of hepatoma-bearing rats coincides with the loss of the enzyme from the hepatoma plasma membrane. Experientia 42, $826-828$.

Dr. Stefan Eggstein

Chirurgische Üniversitätsklinik

Hugstetterstraße 55

D-7800 Freiburg 